\title{
Primary Progressive Aphasia: Toward a Pathophysiological Synthesis
}

\author{
Justina Ruksenaite ${ }^{1} \cdot$ Anna Volkmer $^{2}$. Jessica Jiang ${ }^{1} \cdot$ Jeremy CS Johnson ${ }^{1} \cdot$ Charles R Marshall $^{3}$ - Jason D Warren ${ }^{1}$. \\ Chris JD Hardy ${ }^{1}$
}

Accepted: 13 January 2021 / Published online: 4 February 2021

(C) The Author(s) 2021

\begin{abstract}
Purpose of Review The term primary progressive aphasia (PPA) refers to a diverse group of dementias that present with prominent and early problems with speech and language. They present considerable challenges to clinicians and researchers. Recent Findings Here, we review critical issues around diagnosis of the three major PPA variants (semantic variant PPA, nonfluent/agrammatic variant PPA, logopenic variant PPA), as well as considering 'fragmentary' syndromes. We next consider issues around assessing disease stage, before discussing physiological phenotyping of proteinopathies across the PPA spectrum. We also review evidence for core central auditory impairments in PPA, outline critical challenges associated with treatment, discuss pathophysiological features of each major PPA variant, and conclude with thoughts on key challenges that remain to be addressed.

Summary New findings elucidating the pathophysiology of PPA represent a major step forward in our understanding of these diseases, with implications for diagnosis, care, management, and therapies.
\end{abstract}

Keywords Primary progressive aphasia $\cdot$ Frontotemporal dementia $\cdot$ Alzheimer's disease $\cdot$ Logopenic aphasia $\cdot$ Semantic dementia $\cdot$ Progressive nonfluent aphasia $\cdot$ Physiology

\section{Introduction: the Scope of the Problem}

The language-led dementias or 'primary progressive aphasias' (PPA) are a unique group of neurodegenerative proteinopathies that share a propensity to target language networks of the human brain, with symptom onset often occurring before the age of 65 . Although clinical disorders that would now be termed PPA have been accurately described since the nineteenth century, only in the last few decades have

Jason D Warren and Chris JD Hardy contributed equally to this work.

This article is part of the Topical Collection on Behavior

Chris JD Hardy

chris.hardy@ucl.ac.uk

1 Dementia Research Centre, Department of Neurodegenerative Disease, UCL Queen Square Institute of Neurology, University College London, 8 - 11 Queen Square, London WC1N 3BG, UK

2 Division of Psychology and Language Sciences, University College London, London, UK

3 Preventive Neurology Unit, Wolfson Institute of Preventive Medicine, Queen Mary University of London, London, UK the complexity and variability of these diseases become widely appreciated [1•]. Arguably more than any other condition, PPA has transformed our picture of neurodegenerative diseases as disorders of selective neural vulnerability and targeted network disintegration [2, 3]. However, despite considerable progress in detailing the clinical features, structural brain anatomy, and histopathology of PPA, many challenges remain. The current (2011) consensus criteria for syndromic diagnosis based on clinical, neuropsychological, and neuroimaging features [4] do not capture a significant proportion of the clinical spectrum [5]. We lack a clinical staging system capturing verbal and non-verbal symptoms across the main PPA variants; or robust, dynamic biomarkers of disease progression and prognosis. The associations between pathogenic protein deposition and clinical phenotypes are poorly defined; and above all, effective treatments for preserving day-to-day functioning and modifying disease course have not yet been developed. These issues are crucial not only for people living with PPA but as a paradigm for similar challenges spanning the range of neurodegenerative disease.

Here, we argue that these problems might be addressed by an improved pathophysiological understanding of PPA. In 
support of our argument, we consider recent developments in the field to show how a pathophysiological paradigm might inform clinical and molecular characterisation, prognosis, novel biomarker development, and therapies in PPA.

\section{The Challenge of Clinical Diagnosis: Detection and Definition}

Early and accurate diagnosis of PPA is crucial to allow patients and families to plan for the future and to access the support and services they need. Moreover, it is the gateway to effective management, which is likely to include disease modifying therapies in the foreseeable future. Currently, three main clinico-anatomical syndromes of PPA are recognised in consensus diagnostic criteria $[1 \bullet, 4]$. First, nonfluent/ agrammatic variant PPA (nfvPPA), characterised by insidious impairment of speech sound and connected speech production and subsequently other language output channels, associated with dysfunction and atrophy predominantly involving left peri-Sylvian cortices centred on inferior frontal gyrus and anterior insula [1•]. Second, semantic variant PPA (svPPA), characterised by erosion of knowledge about words, and ultimately objects and concepts across all sensory modalities, associated with dysfunction and atrophy of the semantic appraisal network, most severe in antero-mesial temporal lobe and generally initially predominantly left-sided $[1 \bullet, 6]$. Third, logopenic variant PPA (lvPPA), characterised by progressive anomia and phonological processing, in particular auditory verbal working memory, associated with dysfunction and atrophy usually predominantly involving left temporo-parietal cortices [7]. Representative coronal sections of T1-weighted MRI scans for each major PPA subtype are shown in Fig. 1.

Applying these diagnostic criteria represents a significant challenge, even for experts. We have previously produced a 'diagnostic roadmap' to help the decision-making process [1•]. However, whilst assessment of some domains (e.g. naming, repetition) can be achieved with relative ease, other components such as assessing apraxia of speech, object knowledge, and 'frank' agrammatism are harder to operationalise [1•]. Whilst semantic variant PPA is widely recognised as a coherent diagnostic category [8], fragmentary syndromes fall under the umbrellas of nfvPPA and lvPPA. Within nfvPPA, there may be a distinct group of people with 'pure' apraxia of speech [9] (which can itself be fractionated into phonetic, prosodic, and mixed subvariants [10]), and cases of pure agrammatism have also been identified [11•]. Primary progressive dynamic aphasia has also been reported as a subvariant of nfvPPA [12,13], and several cases of nfvPPA with prominent auditory processing symptoms have also been identified (see section below). In logopenic variant PPA, research has identified three different subvariants based on core deficits of (i) single-word comprehension, (ii) repetition, and (iii) confrontation naming, each associated with specific atrophy profiles [14•]. Moreover, vocabulary loss (a core feature of svPPA) may be relatively common in nfvPPA [15]. A lack of understanding of the underlying pathophysiology in PPA syndromes is a common theme that makes principled diagnostic classification difficult. These difficulties with clinical differentiation of PPA variants have led to the suggestion that common syndromes across the frontotemporal dementia (FTD) spectrum (including nfvPPA and svPPA) are not discrete in their clinical features, existing in a multidimensional space [8•]: according to this formulation, diagnostic criteria for PPA may need to be broadened to include intermediate diagnoses [16].

Whilst typically being regarded as young-onset dementias, recent epidemiological evidence suggests that there is a cluster of older people in their eighth or ninth decade who develop nfvPPA [17••]: this group is likely to be significantly under-diagnosed. There can also be significant problems with symptom onset: whilst the major PPA variants can initially present as selective deficits in specific domains, over time, a 'mixed' phenotype is likely to emerge. This mixed phenotype may, however, be present from the initial stages of the disease [18], creating considerable diagnostic challenges. The
SVPPA

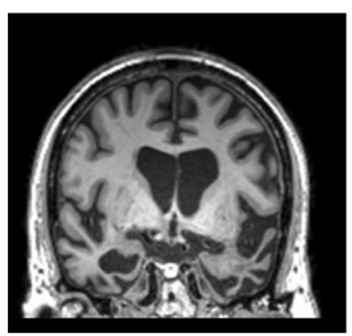

Fig. 1 Neuroanatomical profiles of the major syndromes of primary progressive aphasia. Panels present T1-weighted coronal brain MRI sections of patients with typical syndromes of semantic variant primary progressive aphasia (svPPA), nonfluent/agrammatic primary progressive aphasia (nfvPPA), and logopenic variant primary progressive aphasia (lvPPA). Brain images are presented with the left hemisphere on the
nfvPPA

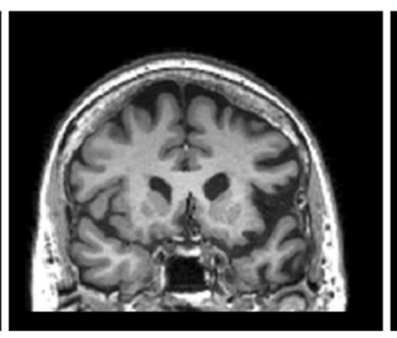

right. The svPPA scan shows asymmetric (predominantly left-sided) anterior inferior and mesial temporal lobe atrophy; the nfvPPA scan shows asymmetric (predominantly left-sided) inferior frontal, insular, and anterior-superior temporal gyrus atrophy, while the lvPPA scan shows asymmetric (predominantly left-sided) temporo-parietal junction atrophy 
opposite problem is true too: leading symptoms in all PPA variants can be extremely subtle and are not always well captured by the current criteria, which focus on established disease [4]. Moreover, despite being characterised as 'languageled' disorders, patients and carers often report extra-linguistic features, for which supporting research evidence is now emerging, including auditory dysfunction (see section below) and abnormal nonverbal behaviours [19, 20]. Given that early detection and diagnosis is likely to be vital for successful therapies, a reformulation of the criteria around identification of the very earliest symptoms would be timely.

\section{The Challenge of Clinical Prognosis: Assessing Disease Stage and Activity}

Prognosis and anticipation of deficits later on are hugely important for patients and carers, yet robust and reliable ways of assessing disease stage and rate of progression are currently lacking. Rates of clinical evolution differ between the major PPA variants and may be more rapid in nfvPPA [21]. A significant proportion of people with nfvPPA will develop Parkinsonism, which seems more likely to develop in those people who have prominent apraxia of speech [22, 23••], but clear early pathophysiological markers of anticipated trajectories across the PPA spectrum are needed. A related issue is the lack of widely applicable severity measures across PPA syndromes: 'standard' tools are heavily focused on symptoms associated with typical Alzheimer's disease and may significantly over-emphasise severity in PPA due to heavy language components, as with the Mini-Mental State Examination, whilst the Frontotemporal Dementia Rating Scale [24] was not developed for people with lvPPA, and the Progressive Aphasia Severity Scale [25] does not take account of nonlanguage symptoms. The recently developed Mini Linguistic State Examination [26•] may help to address some of these issues, but there remains a clear need for a symptom-led staging system for each of the major PPA variants that can capture the range of language and non-verbal symptoms that are present in each subtype [1•].

Evidence suggests a clear rationale for a personalised approach: language functions are highly dependent on life trajectory, and intriguing links have been identified between PPA and dyslexia [27]. Very recent work suggests that bilingualism may delay the onset of lvPPA [28]. Much needs to be done in identifying the earliest symptoms of PPA: in svPPA, for example, the patient will virtually always have established focal atrophy on MRI at the time of diagnosis, and standard psychometric tests are often subject to floor/ceiling effects: svPPA patients typically struggle to name more than a single item correctly on the graded naming test [29••]. Identification and stratification into clinical trials at the very earliest stage of disease will be vital if treatments are to be effective. The use of ecologically relevant measures that quantify aspects of speech output have garnered recent interest in terms of both diagnosis and disease tracking from early stages [30-33], leading to the suggestion that automated analysis of speech might be used as a 'verbal thermometer' for PPA and FTD [34]. There is also considerable interest in the use of 'wet biomarkers' in measuring disease burden and intensity. For instance, neurofilament light chain protein has emerged as a measure of disease intensity across the FTD spectrum, regardless of the underlying pathology [35]. However, despite these strides forward in tracking biomarkers of disease intensity, information is currently lacking about symptom management, quality of life, and palliative care in late-stage PPA.

\section{The Challenge of Molecular Diagnosis: Physiological Phenotyping of Proteinopathies}

One of the most ambitious, but most important, challenges in neurodegenerative disease research is to identify how pathogenic proteins give rise to complex phenotypes. The major molecular histopathological associations differ between the major syndromes of PPA [22, 23••, 36, 37•]: nfvPPA is most often associated with primary tauopathies, svPPA is closely associated with TDP-43 (type C) pathology, and lvPPA with Alzheimer pathology, leading to the proposal that the PPA may constitute 'molecular nexopathies' [2], i.e. specific conjunctions of macroscopic network characteristics and pathogenic protein properties. This formulation has received empirical support [22, 38, 39]. However, there is not complete concordance between phenotype and pathology: svPPA can more rarely be caused by tau or Alzheimer pathologies [22, 37•, 40]; nfvPPA is sometimes caused by Alzheimer's disease, or TDP43 (type A or B) [22, 37•, 41, 42]; lvPPA has been associated with dementia with Lewy bodies[43] and TDP-43 (type A) [41]; and an lvPPA-like phenotype has been consistently identified in people with mutations in the progranulin gene [44, 45]. Emerging evidence suggests that specific language network vulnerabilities caused by genetic, developmental, and/or lifestyle factors may determine why some people develop a PPA phenotype in the context of a specific proteinopathy [27, $46,47]$. Age at onset may also influence phenotypic expression [48]. Clinical and neuropsychological tests may reveal certain clues as to the underlying proteinopathy in PPA: non-verbal episodic memory deficits are associated with Alzheimer pathology [49], whilst Parkinsonism (often evolving into a progressive supranuclear palsy/corticobasal syndrome) and apraxia of speech are typically associated with a tauopathy [22, 23••]. Motor neuron disease features accompanying nfvPPA and svPPA may be under-recognised and predict underlying histopathology [50, 51].

Whilst most PPA syndromes are sporadic, making presymptomatic diagnosis difficult, rarer cases of progranulin- 
associated aphasia [44, 45] and other genetic forms of PPA could represent important 'test' cases. Communication functions are uniquely complex, and carefully designed tests that can tax the integrity of these vulnerable networks might be used as 'stress tests' of early disease. In PPA, paradigms of artificially degraded speech may hold promise in this regard [52••], as tests of degraded visual object processing have emerged as early markers of cognitive decline in the context of Lewy body disease [53]. Other novel tests probing altered socio-emotional reactivity $[54,55]$, autonomic functions [56, $57 \cdot \bullet$ ], sleep symptoms [58], and other functions may also track protein-linked dysfunction across a wider range of disease stages than conventional psychology tests.

A current major focus is in the use of in vivo positron emission tomography imaging. However, whilst there have been promising developments with radioligands showing increased binding to pathologically affected regions in tauopathies, the specificity of these ligands is typically unsatisfactory [59]. Developments are ongoing to develop ligands that may bind with greater specificity to tau and TDP-43, whilst markers of neuroinflammation may represent promising tools for tracking disease severity regardless of proteinopathy [60,61]. Novel, connectivity-based analysis approaches may help to elucidate mechanisms of pathogenic protein spread: recent work using spectral dynamic causal modelling has suggested that attenuation of inhibitory connectivity in antero-mesial temporal lobes may help drive TDP-43 (type C) pathogenic protein spread in svPPA[62••]. Innovative magnetoencephalography [63-66] and functional magnetic resonance imaging $[29 \bullet \bullet, 54]$ paradigms have also shown utility in delineating unique neurophysiological signatures of functional connectivity and plasticity in major PPA variants: these techniques may hold promise as very early markers of neurodegeneration, when atrophy is not prominent [67].

\section{Evidence of Central Auditory Impairment on Psychoacoustic Tests}

Language and auditory functions are closely interrelated [68], meaning that auditory measures may represent useful probes of integrity of the relevant networks in PPA [69]. Indeed, language output deficits in all three major PPA syndromes are likely to be significantly influenced by disordered complex sound processing and understanding: an emerging picture in PPA is of fundamental deficits in central auditory perception $[70,71]$. People with svPPA show profound environmental sound agnosia [71] and phonagnosia [72], alongside symptoms of tinnitus and hyperacusis [73]. Deficits at a relatively 'early' level of auditory processing have been identified in nfvPPA, including sound detection [74••]; reduced activity in primary auditory cortex[29••]; pitch and timbral pattern processing [71]; and processing of rhythmicity of tone sequences $[29 \bullet \bullet, 75 \bullet \bullet, 76]$. Accurate speech perception relies on successful integration of bottom-up sensory information with top-down predictive processing, which is also thought to be impaired in nfvPPA [77•]. Logopenic variant PPA is associated with deficient processing of phonemes [29••, $78 \cdot \bullet$ ]. One parsimonious explanation for the phonemic errors made in speech output in lvPPA is that these relate to a general impairment in phonemic representation, though the association between phonemic input and output errors is yet to be explored experimentally. Patients with lvPPA also show pronounced difficulties understanding degraded speech [52・•], which may reflect a more general deficit in terms of parsing the auditory environment ('auditory scene analysis') - this has not been tested experimentally but accords with findings in typical Alzheimer's disease and posterior cortical atrophy (a visuospatial form of Alzheimer's disease) suggesting that damage to posteromedial cortices may underpin these deficits across the $\mathrm{AD}$ spectrum $[79,80]$.

Atypical presentations in the PPA/FTD spectrum manifesting as very early problems with auditory processing have also been identified, including progressive pure phonagnosia [46, 81], progressive word deafness [82-84], and generalised auditory agnosia [85]. Taken together, these recent findings suggest that the PPA might be characterised as pervasive 'communication' disorders that go beyond language. Tests of auditory processing could have considerable advantages over tests measuring language functions: they are relatively easy to measure and administer, and less linguistically/culturebound than language tests, making them attractive as outcome measurements in future clinical trials.

\section{The Challenge of Treatment: Optimising Function And Changing Disease Course}

Unfortunately, no disease-modifying treatments for any PPA variant currently exist, though clinical trials are now underway for tauopathies (for a review, see[86]). Disease modification approaches will necessarily be protein-directed, rather than PPA specific, re-emphasising the need for some kind of molecular biomarker. Physiological understanding may improve prediction of molecular pathology, but it is also important for monitoring response to treatment and demonstrating efficacy in these syndromes specifically. Without disease-modifying therapies, non-pharmacological approaches to PPA management are of considerable importance, and non-invasive brain stimulation techniques to ameliorate specific symptoms across PPA variants have received much recent attention. Transcranial direct current stimulation as an adjunct to traditional speech and language therapy has shown some promise in PPA [87, 88], and there have been some reports of success with transcranial magnetic stimulation in case studies and small cohorts [89-91]; clinical trials are currently underway. 
People with PPA may benefit from physiologically informed cognitive rehabilitation strategies, akin to strategies designed to enhance neuroplasticity after stroke aphasia [92, 93]: recent work suggests that people with all major forms of PPA have retained capacity for perceptual learning of degraded speech [52*0], and that patients with nfvPPA show preserved faculty for artificial grammar learning [94], suggesting the need for future trials focused on exposure-based approaches to rehabilitation of agrammatism and degraded speech perception. In svPPA, right-lateralised brain regions show elevated activity in magnetoencephalography when listening to spoken words, whilst dorsal regions appear to compensate for damaged ventral regions when patients read irregular words, together suggesting a degree of functional plasticity in brain networks with relatively preserved integrity $[63,64]$.

A 'one size fits all' approach is unlikely to be effective in PPA, and prevailing research suggests that pathophysiologically targeted speech and language therapy interventions may yield most success $[95,96]$. In svPPA, improvement in vocabulary after naming intervention has been observed alongside activation of larger networks in bilateral brain regions on functional neuroimaging [97-99, 100••, 101], though word-retrieval therapies are only likely to yield benefit on trained items (transfer to untrained items is more likely to be seen in lvPPA [101]). In nfvPPA, script training has been shown to improve automation of speech production, resulting in immediate and long-term outcomes in terms of intelligibility and grammaticality, up to 1 year after treatment [102].

In recent years, there has been an increase in research focusing on compensatory, or functional approaches to symptom management for people with PPA [103]. These approaches focus on compensating for the speech and language difficulties through use of strategies, environmental supports, or augmentative alternative communication aids. This reflects the practice of clinical speech and language therapists who report prioritising communication partner training approaches when with people with PPA and their family members [104-107]. This approach targets everyday conversation between a person with PPA and a family member or carer, and is underpinned by an assessment of strategies that facilitate communication (e.g. gesture) and those that act as barriers (e.g. completing a person's sentence without an invitation to do so, or abrupt topic changes) [105, 108].

Despite this emerging evidence base, speech and language therapists across the UK report barriers to people with PPA accessing their services including a lack of knowledge amongst referrers about the benefits of speech and language therapy for PPA, restrictive service criteria and commissioning limitations, and a lack of confidence [106, 107]. These issues are reflected internationally, with similar issues reported across the USA and Australia [105], highlighting a need for further work in developing the relevant research evidence to underpin care pathways to support referrers, with a complementary focus on education of health care professionals (including speech and language therapists) and commissioners. Dedicated PPA support groups have an important role to play in comprehensive PPA care [109]. In the UK, Rare Dementia Support run the national PPA Support Group (www.raredementiasupport.org) and there are many others in different countries.

\section{An Interim Pathophysiological Synthesis of PPA}

We argue that the rich phenomenology of PPA is underpinned by core pathophysiological processes that differ between canonical PPA syndromes (diagrammed schematically in Fig. 2).

The motivation for moving beyond traditional neurolinguistics accounts of these syndromes to address core pathophysiological mechanisms is fourfold. Firstly, a pathophysiological perspective may explain certain associated clinical features of PPA syndromes (such as nonverbal auditory dysfunction and complex nonverbal behavioural changes) that are difficult to reconcile with purely neurolinguistic accounts. Secondly, it grounds PPA in neurobiological mechanisms of language that emphasise neural circuit function (see for example [110]), thereby building bridges to the molecular pathological processes that target these circuits and potentially linking complex disease phenotypes to the activity of pathogenic proteins. Thirdly, it opens up fresh avenues for characterising these diseases using physiologically informed methods, such as animal models, artificial neural networks, and functional neuroimaging. Finally, it promises to motivate the development of new, cross-linguistic, physiological biomarkers for detecting and tracking the effects of culprit pathogenic proteins and therapeutic interventions dynamically and ultimately, to inform the design of novel therapies.

\section{Features of Language Networks Confer Susceptibility to Neurodegenerative Pathologies}

Dynamic transformation of information (from percept to meaning and to action) is integral to the normal operation of the language system. This was implicit in the early models of Lichtheim and others but modern neuroimaging and neurophysiological techniques have elaborated candidate neural mechanisms by which linguistic transformation occurs-in particular, predictive coding and matching of sensory stimuli and motor programmes to stored neural representations or 'templates', operating iteratively across hierarchically organised neural circuits $[110,111]$.

The transformation of speech signals engages highly distributed neural networks and is characterised neurophysiologically by spatio-temporal integration, nonlinear coding, plasticity, and reciprocal interaction between processing stages. These are generic neurophysiological processes that also operate on 


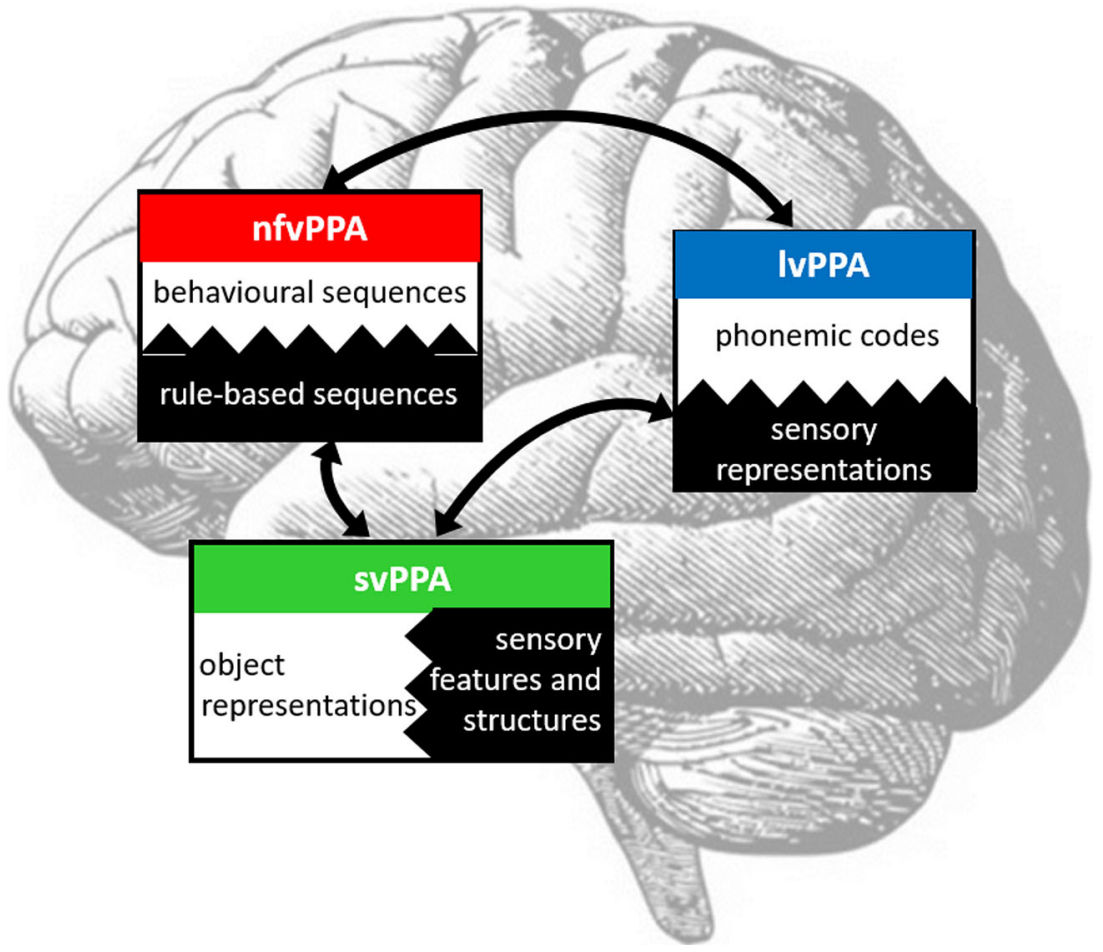

Fig. 2 Proposed pathophysiological synthesis of primary progressive aphasias. The figure diagrams core neural processes proposed to be targeted in each of the canonical syndromes of primary progressive aphasia, projected on a lateral view of the left cerebral hemisphere. Oblongs signify core neural processing modules or circuits within the language network: each instantiates a key template-matching operation in which incoming data (represented by black hatching) is iteratively reconciled with prior predictions and transformed into an output (predictive decoding; see text). The bidirectional arrows represent the reciprocal exchange of data and predictions between core processing modules. Processing modules are organised hierarchically, in that incoming sensory representations arriving at temporoparietal junctional cortex (blue) are transformed into increasingly abstract conceptual representations in anterior temporal cortex (green) and may ultimately be used in generating a motor output via anterior peri-Sylvian mechanisms (red). However, extensive dynamic interactions between processing modules are essential to the normal operation of the language system. Note that the neuroanatomical loci of the processing modules designated here correspond only loosely to 'Broca's area', 'Wernicke's area', and other structures emphasised in classical (vascular) aphasiology; the primary progressive aphasia are essentially network-based disorders. lvPPA, logopenic variant of primary progressive aphasia; nfvPPA, nonfluent-agrammatic variant of primary progressive aphasia; svPPA, semantic variant of primary progressive aphasia

elements, in line with the molecular nexopathies paradigm [2, 3] (TDP-43 in semantic appraisal network, svPPA; AD pathology in default mode network, lvPPA; hyperphosphorylated tau in dorsal peri-Sylvian networks, nfvPPA). Following this formulation, the greater clinical and pathological heterogeneity of nfvPPA might reflect the lack of a single coherent 'nexopathy' in this syndrome. Certain molecular pathologies may affect more than one core neural process from an early stage, leading to atypical or mixed PPA phenotypes (for example, progranulin mutations may produce widespread degeneration of long-range pathways within the language hemisphere [45]).

\section{SVPPA}

This syndrome has been more widely studied from a pathophysiological perspective than other PPA syndromes. A candidate generic mechanism for svPPA is impaired computation of the featural statistics that support categorisation and identification of sensory stimuli, due to degradation of the neural activity patterns 
corresponding to coherent object and concept templates. This mechanism is suggested by several converging lines of evidence. Neuropsychologically, patients show impaired recognition of idiosyncratic exemplars and inappropriate generalisation between object categories due to over-reliance on superficial sensory (rather than conceptual) similarities, in both verbal and nonverbal domains (e.g. they may misclassify a Manx cat as a dog, or sound the English word sew as 'soo' [6, 112]), but also show impaired ability to process higher-order regularities in sensory stimuli such as syllable strings [29・•, 76] and may have difficulty distinguishing 'real' objects from comparably perceptually complex foils [71]. Neurophysiologically, there is reduced GABAergic recurrent inhibition within local circuits of the core semantic appraisal network $[62 \cdot \bullet, 113]$, predicting a loss of definition of stored neural object templates and erosion of boundaries between object representations. The complex behavioural changes associated with svPPA such as rigidity, disinhibition, and altered dietary preferences might be in part compensatory but could equally reflect altered processing of socio-emotional signals: indeed, behavioural disturbance in svPPA has a neurophysiological substrate in common with impaired object recognition [62・•].

\section{IvPPA}

The core mechanism of this syndrome may be impaired activation of phonemic templates, leading to deficient parsing of input sensory signals for transcoding to phonological output. Such a mechanism might account for a number of core features of the syndrome, including anomia, phonological, and neologistic errors in speech and writing and reduced verbal working memory capacity $[1 \bullet, 7,114-117]$. It could also plausibly underpin recent findings of impaired understanding of degraded speech $[52 \cdot \bullet]$ and impaired phonemic discrimination [78.0] in lvPPA. This mechanism might further account for various bedside observations that are less well characterised. For example, on a phrase repetition task, patients tend to demonstrate not merely a reduced verbal working memory span, but intrusions from previously administered phrases and 'hunting' after the correct item via a series of approximations, suggesting impaired 'refreshing' of the verbal buffer. In addition, patients commonly show tip-of-the-tongue phenomena, consistent with impaired activation of phonological representations $[1 \bullet, 118,119]$ and - to the extent that initial activation of word representations is necessary to access the verbal semantic system [120] - this mechanism might also contribute to the variable semantic deficit in lvPPA [114, 121]. There is some direct fMRI evidence for impaired activation of phonological representations in lvPPA linked to involvement of temporo-parietal junctional cortex [29••], which is both the key locus of auditory-motor phonological transformations in the healthy brain [122], and a primary target of the pathological process in lvPPA $[7,14 \cdot, 118]$. Although variability of deficits between series may point to separate sub-syndromes within lvPPA [14•], it is possible that this variation is at least in part physiologically based, reflecting the dynamic impact of Alzheimer pathology (the most frequent molecular substrate) on modulatory cholinergic transmission [123].

\section{nfvPPA}

This syndrome is typically considered a predominantly motor disorder of impaired speech and language output and it remains unclear whether there is a 'pure' motor sub-syndrome within the nfvPPA spectrum $[1 \bullet, 4,9,10,114,124]$. However, many if not most patients evolve associated impairments of linguistic processing, whilst the hallmark feature of expressive agrammatism in general signifies a more pervasive disorder of sentence processing [114]. Verbal working memory is also frequently impaired [114]. A candidate, generic unifying mechanism may be impaired temporal scaffolding and/or combinatorial sequencing and scheduling of speech and other sensori-motor routines, particularly where these depend on predictive coding governed by learned 'rules'. Such a mechanism might underpin the more basic impairments of musical, prosodic, and other auditory pattern analysis recently described in nfvPPA, notably affecting the discrimination of rhythm and regularity $[29 \bullet \bullet, 33,71,75 \bullet \bullet, 76$, $125,126 \cdot 127,128]$. The difficulty that patients with nfvPPA experience in processing speech and other auditory signals may be at least in part attributable to inflexible neural predictions about incoming sensory information and delayed updating of neural templates based on errors [77॰]. Such a deficit might account for other, diverse phenomena documented in nfvPPA that on face value are difficult to reconcile with an essentially motoric disorder. These include impaired pure tone perception [74••], degraded speech comprehension [52••], and, in patients' spontaneous speech, the frequent appearance of 'binary reversals' (substitution of closely related words with incorrect polarity, e.g. 'yes' for 'no') [129]. This pathophysiological formulation accords with neuroanatomical evidence implicating dorsal anterior cingulate and supplementary motor cortex in the impaired analysis of temporal patterns in nfvPPA [29, 76, 127]. More broadly, it fits with the well-documented role played by inferior frontal gyrus, anterior superior temporal gyrus, and anterior insula (the core cortical targets of nfvPPA) in the hierarchical processing of linguistic and non-linguistic sequences [130-135] and the intimate, oscillatory interaction between top-down and bottom-up mechanisms during speech processing [136-138].

\section{Conclusions and Future Directions}

Here, we have outlined recent progress toward a pathophysiology of PPA syndromes. Improved physiological understanding may improve the in vivo prediction of molecular pathologies, improve our ability to monitor treatment response and demonstrate efficacy, and improve the design of targeted 
symptomatic interventions. These interventions include "traditional' speech and language therapy approaches, but auditory rehabilitation strategies may also hold promise in ameliorating some of the core auditory symptoms outlined above. Despite encouraging recent progress, there are a number of issues that remain to be addressed. First, much work remains to be done in defining new pathophysiological markers of these diseases - progress will depend on an integrated multimodality approach including functional neuroimaging, neurophysiological, and histopathological techniques, not merely to recapitulate well-established patterns of network disintegration but to identify novel markers of neural reorganisation and plasticity [139]. Second, PPA is rare-if we are to be successful in defining coherent clinico-pathological entities, we will need larger cohorts of patients, which will require large multicentre studies using instruments that are appropriate for speakers of different languages where possible (tests of basic auditory processing may be an attractive prospect here). Third, a validated symptom-led staging system for the major forms of PPA will be vital for tracking disease evolution and planning appropriate care pathways. Finally, the Covid-19 pandemic has changed all of our lives in unprecedented ways, and people with PPA and their caregivers face unique challenges $[140 \bullet \bullet$. The almost universal social dislocations imposed by the virus will ideally motivate clinicians and health care providers to take advantage of the innovative technological ways of working that have been developed: evidence suggests that teletherapy is possible within the context of PPA [141], and perhaps remote- and videoconferencing-based assessments could pave the way for the future national and international collaborations that are needed. Finally, we believe that it may now be time to update the current PPA consensus criteria, taking into account a decade of new research findings and incorporating physiologically informed disease metrics.

Funding The Dementia Research Centre is supported by Alzheimer's Research UK, Brain Research Trust, and The Wolfson Foundation. This work was supported by the Alzheimer's Society, Alzheimer's Research UK, and the National Institute for Health Research University College London Hospitals Biomedical Research Centre. JJ is supported by a Frontotemporal Dementia Research Studentship in Memory of David Blechner (funded through The National Brain Appeal). JCSJ is supported by an Association of British Neurologists Clinical Research Training Fellowship. CRM is supported by a grant from Bart's Charity. CJDH is supported by a RNID-Dunhill Medical Trust Pauline Ashley Fellowship (PA23_Hardy). AV is funded by a National Institute for Health Research Development Skills Enhancement Award. This paper presents independent research funded by the National Institute for Health Research (NIHR). The views expressed are those of the author(s) and not necessarily those of the NHS, the NIHR, or the Department of Health and Social Care.

\section{Declarations}

Conflict of Interest The authors declare that they have no conflict of interest.
Human and Animal Rights and Informed Consent This article does not contain any studies with human or animal subjects performed by any of the authors.

Open Access This article is licensed under a Creative Commons Attribution 4.0 International License, which permits use, sharing, adaptation, distribution and reproduction in any medium or format, as long as you give appropriate credit to the original author(s) and the source, provide a link to the Creative Commons licence, and indicate if changes were made. The images or other third party material in this article are included in the article's Creative Commons licence, unless indicated otherwise in a credit line to the material. If material is not included in the article's Creative Commons licence and your intended use is not permitted by statutory regulation or exceeds the permitted use, you will need to obtain permission directly from the copyright holder. To view a copy of this licence, visit http://creativecommons.org/licenses/by/4.0/.

\section{References}

Papers of particular interest, published recently, have been highlighted as:

- Of importance

•. Of major importance

1. Marshall CR, Hardy CJD, Volkmer A, Russell LL, Bond RL, Fletcher PD, et al. Primary progressive aphasia: a clinical approach. J Neurol. 2018;265:1474-90. This recent review article includes observations of less widely known symptoms of PPA, and outlines a clinical roadmap for bedside assessment of PPA.

2. Warren JD, Rohrer JD, Schott JM, Fox NC, Hardy J, Rossor MN. Molecular nexopathies: a new paradigm of neurodegenerative disease. Trends Neurosci Elsevier Ltd. 2013;36:561-9.

3. Tee BL, Gorno-Tempini ML. Primary progressive aphasia: a model for neurodegenerative disease. Curr Opin Neurol Lippincott Williams and Wilkins. 2019:255-65.

4. Gorno-Tempini ML, Hillis AE, Weintraub S, Kertesz A, Mendez M, Cappa SF, et al. Classification of primary progressive aphasia and its variants. Neurology American Academy of Neurology. 2011;76:1006-14.

5. Sajjadi SA, Patterson K, Arnold RJ, Watson PC, Nestor PJ. Primary progressive aphasia: a tale of two syndromes and the rest. Neurology American Academy of Neurology. 2012;78:1670-7.

6. Hodges J, Patterson K. Semantic dementia: a unique clinicopathological syndrome. Lancet Neurol. 2007;6:3-8.

7. Rohrer JD, Ridgway GR, Crutch SJ, Hailstone J, Goll JC, Clarkson MJ, et al. Progressive logopenic/phonological aphasia: erosion of the language network. Neuroimage Elsevier. 2010;49:984-93.

8. Ingram RU, Halai AD, Pobric G, Sajjadi S, Patterson K, Ralph MAL. Graded, multidimensional intra- and intergroup variations in primary progressive aphasia and post-stroke aphasia. Brain Oxford University Press (OUP). 2020; The authors report on a novel principal component analysis (PCA) approach to classification, revealing SvPPA to be a 'true' diagnostic category, with overlap and graded variations between other progressive and post-stroke aphasias.

9. Josephs K, Duffy J, Strand E, Machulda M. Characterizing a neurodegenerative syndrome: primary progressive apraxia of speech. Brain. 2012;135:1522-36.

10. Josephs KA, Duffy JR, Strand EA, Machulda MM, Senjem ML, Lowe VJ, et al. Syndromes dominated by apraxia of speech show 
distinct characteristics from agrammatic PPA. Neurology. 2013;81:337-45.

11. Tetzloff KA, Duffy JR, Clark HM, Utianski RL, Strand EA, Machulda MM, et al. Progressive agrammatic aphasia without apraxia of speech as a distinct syndrome. Brain Oxford University Press. 2019;142:2466-82. This study presents a comprehensive characterization of progressive agrammatic aphasia without apraxia of speech as a distinct clinicopathological syndrome.

12. Warren JD, Warren JE, Fox NC, Warrington EK. Nothing to say, something to sing: primary progressive dynamic aphasia. Neurocase. 2003;9:140-55.

13. Perez DL, Dickerson BC, McGinnis SM, Sapolsky D, Johnson K, Searl M, et al. You don't say: dynamic aphasia, another variant of primary progressive aphasia? J Alzheimer's Dis IOS Press. 2013;34:139-44.

14. Leyton CE, Hodges JR, McLean CA, Kril JJ, Piguet O, Ballard KJ. Is the logopenic-variant of primary progressive aphasia a unitary disorder? Cortex Elsevier. 2015;67:122-33. The authors outline issues of variability within the IvPPA spectrum, suggesting that there may be three distinct IvPPA subtypes, with dissociable cognitive and neuroanatomical profiles.

15. Schaeverbeke J, Gabel S, Meersmans K, Bruffaerts R, Liuzzi AG, Evenepoel C, et al. Single-word comprehension deficits in the nonfluent variant of primary progressive aphasia. Alzheimer's Res Ther BioMed Central Ltd. 2018;10:68.

16. Murley AG, Coyle-Gilchrist I, Rouse MA, Jones PS, Li W, Wiggins J, et al. Redefining the multidimensional clinical phenotypes of frontotemporal lobar degeneration syndromes. Brain NLM (Medline). 2020;143:1555-71.

17.• Coyle-Gilchrist ITS, Dick KM, Patterson K, Rodríquez PV, Wehmann E, Wilcox A, et al. Prevalence, characteristics, and survival of frontotemporal lobar degeneration syndromes. Neurology Lippincott Williams and Wilkins. 2016;86:1736-43.

18. Mesulam M-M, Wieneke C, Thompson C, Rogalski E, Weintraub S. Quantitative classification of primary progressive aphasia at early and mild impairment stages. Brain. 2012;135:1537-53.

19. Singh TD, Duffy JR, Strand EA, Machulda MM, Whitwell JL, Josephs KA. Neuropsychiatric symptoms in primary progressive aphasia and apraxia of speech. Dement Geriatr Cogn Disord. 2015;39:228-38.

20. Van Langenhove T, Leyton CE, Piguet O, Hodges JR. Comparing longitudinal behavior changes in the primary progressive aphasias. J Alzheimer's Dis IOS Press. 2016;53:1033-42.

21. Sebastian R, Thompson CB, Wang NY, Wright A, Meyer A, Friedman RB, et al. Patterns of decline in naming and semantic knowledge in primary progressive aphasia. Aphasiology Routledge. 2018;32:1010-30.

22. Rohrer JD, Lashley T, Schott JM, Warren JE, Mead S, Isaacs AM, et al. Clinical and neuroanatomical signatures of tissue pathology in frontotemporal lobar degeneration. Brain. 2011;134:2565-81.

23.• Spinelli EG, Mandelli ML, Miller ZA, Santos-Santos MA, Wilson SM, Agosta F, et al. Typical and atypical pathology in primary progressive aphasia variants. Ann Neurol. 2017; The authors describe histopathological correlates of PPA in a large, wellcharacterized sample.

24. Mioshi E, Hsieh S, Savage S, Hornberger M, Hodges JR. Clinical staging and disease progression in frontotemporal dementia. Neurology. 2010;74:1591-7.

25. Sapolsky D, Domoto-Reilly K, Dickerson BC. Use of the Progressive Aphasia Severity Scale (PASS) in monitoring speech and language status in PPA. Aphasiology Routledge. 2014;28:993-1003.

26. Patel N, Peterson KA, Ingram R, Storey I, Cappa SF, Catricala E, et al. The Mini Linguistic State Examination (MLSE): a brief but accurate assessment tool for classifying Primary Progressive Aphasias. medRxiv. Cold Spring Harbor Laboratory Press. 2020;
The authors introduce the MLSE, a translatable general diagnostic instrument for PPA. This is an important contribution to the field given the lack of such instruments currently.

27. Miller ZA, Mandelli ML, Rankin KP, Henry ML, Babiak MC, Frazier DT, et al. Handedness and language learning disability differentially distribute in progressive aphasia variants. Brain Oxford University Press. 2013;136:3461-73.

28. de Leon J, Grasso SM, Welch A, Miller Z, Shwe W, Rabinovici GD, et al. Effects of bilingualism on age at onset in two clinical Alzheimer's disease variants. Alzheimer's Dement Wiley. 2020: alz.12170.

29.• Hardy CJD, Agustus JL, Marshall CR, Clark CN, Russell LL, Brotherhood EV, et al. Functional neuroanatomy of speech signal decoding in primary progressive aphasias. Neurobiol Aging Elsevier. 2017;56:190-201. The authors use activation fMRI to define fundamental 'building blocks' of speech signal processing in major PPA syndromes.

30. Zimmerer VC, Hardy CJD, Eastman J, Dutta S, Varnet L, Bond RL, et al. Automated profiling of spontaneous speech in primary progressive aphasia and behavioral-variant frontotemporal dementia: an approach based on usage-frequency. Cortex Elsevier. 2020.

31. Nevler N, Ash S, Irwin DJ, Liberman M, Grossman M. Validated automatic speech biomarkers in primary progressive aphasia. Ann Clin Transl Neurol Wiley-Blackwell. 2019;6:4-14.

32. Matias-Guiu JA, Suárez-Coalla P, Pytel V, Cabrera-Martín MN, Moreno-Ramos T, Delgado-Alonso C, et al. Reading prosody in the non-fluent and logopenic variants of primary progressive aphasia. Cortex Masson SpA. 2020;132:63-78.

33. Rohrer JD, Sauter D, Scott S, Rossor MN, Warren JD. Receptive prosody in nonfluent primary progressive aphasias. Cortex Elsevier Srl. 2012;48:308-16.

34. Jarrold W, Rofes A, Wilson S, Pressman P, Stabler E, Gorno-Tempini M. A verbal thermometer for assessing neurodegenerative disease: automated measurement of pronoun and verb ratio from speech. Proc Annu Int Conf IEEE Eng Med Biol Soc EMBS. Institute of Electrical and Electronics Engineers Inc.; 2020. p. 5831-7.

35. Rohrer JD, Woollacott IOC, Dick KM, Brotherhood E, Gordon E, Fellows A, et al. Serum neurofilament light chain protein is a measure of disease intensity in frontotemporal dementia. Neurology. 2016;87:1329-36.

36. Sivasathiaseelan H, Marshall CR, Agustus JL, Benhamou E, Bond RL, Van Leeuwen JEP, et al. Frontotemporal dementia: a clinical review. Semin Neurol Thieme Medical Publishers, Inc. 2019:251-63.

37. Bergeron D, Gorno-Tempini ML, Rabinovici GD, Santos-Santos MA, Seeley W, Miller BL, et al. Prevalence of amyloid- $\beta$ pathology in distinct variants of primary progressive aphasia. Ann Neurol Wiley. 2018;84:729-40. The authors present recent data on amyloid pathology in PPA variants.

38. Giannini LAA, Xie SX, McMillan CT, Liang M, Williams A, Jester $\mathrm{C}$, et al. Divergent patterns of TDP-43 and tau pathologies in primary progressive aphasia. Ann Neurol Wiley. 2019;85:630-43.

39. Borghesani V, Battistella G, Mandelli ML, Welch A, Weis E, Younes $\mathrm{K}$, et al. Regional and hemispheric susceptibility of the temporal lobe to FTLD-TDP type C pathology. NeuroImage Clin Elsevier. 2020;28.

40. Snowden JS, Kobylecki C, Jones M, Thompson JC, Richardson AM, Mann DMA. Association between semantic dementia and progressive supranuclear palsy. J Neurol Neurosurg Psychiatry BMJ Publishing Group. 2019;90:115-7.

41. Mesulam M-M, Weintraub S, Rogalski EJ, Wieneke C, Geula C, Bigio EH. Asymmetry and heterogeneity of Alzheimer's and frontotemporal pathology in primary progressive aphasia. Brain. 2014; 137:1176-92.

42. Ossenkoppele R, Mattsson N, Teunissen CE, Barkhof F, Pijnenburg Y, Scheltens P, et al. Cerebrospinal fluid biomarkers and cerebral atrophy in distinct clinical variants of probable Alzheimer's disease. Neurobiol Aging. 2015;36:2340-7. 
43. Boes S, Botha H, Machulda M, Lowe V, Graff-Radford J, Whitwell JL, et al. Dementia with Lewy bodies presenting as Logopenic variant primary progressive Aphasia. Neurocase Routledge. 2020:1-5.

44. Josephs KA, Duffy JR, Strand EA, Machulda MM, Vemuri P, Senjem ML, et al. Progranulin-associated PiB-negative logopenic primary progressive aphasia. J Neurol Dr Dietrich Steinkopff Verlag GmbH and Co KG. 2014;261:604-14.

45. Rohrer JD, Crutch SJ, Warrington EK, Warren JD. Progranulinassociated primary progressive aphasia: a distinct phenotype? Neuropsychologia. 2010;48:288-97.

46. Didic M, Aglieri V, Tramoni-Nègre E, Ronat L, Le Ber I, Ceccaldi $\mathrm{M}$, et al. Progressive phonagnosia in a telephone operator carrying a C9orf72 expansion. Cortex Elsevier BV. 2020;132:92-8.

47. Weintraub S, Rader B, Coventry C, Sridhar J, Wood J, Guillaume $\mathrm{KA}$, et al. Familial language network vulnerability in primary progressive aphasia. Neurology NLM (Medline). 2020:e847-55.

48. San Lee J, Yoo S, Park S, Kim HJ, Park KC, Seong JK, et al. Differences in neuroimaging features of early- versus late-onset nonfluent/agrammatic primary progressive aphasia. Neurobiol Aging Elsevier Inc. 2020;86:92-101.

49. Ramanan S, Flanagan E, Leyton CE, Villemagne VL, Rowe CC, Hodges JR, et al. Non-verbal episodic memory deficits in primary progressive aphasias are highly predictive of underlying amyloid pathology. J Alzheimer's Dis IOS Press. 2016;51:367-76.

50. Tan RH, Guennewig B, Dobson-Stone C, Kwok JBJ, Kril JJ, Kiernan MC, et al. The underacknowledged PPA-ALS: a unique clinicopathologic subtype with strong heritability. Neurology Lippincott Williams and Wilkins. 2019;92:E1354-66.

51. Vinceti G, Olney N, Mandelli ML, Spina S, Hubbard HI, SantosSantos MA, et al. Primary progressive aphasia and the FTD-MND spectrum disorders: clinical, pathological, and neuroimaging correlates. Amyotroph Lateral Scler Front Degener Taylor and Francis Ltd. 2019;20:146-58.

52.• Hardy CJD, Marshall CR, Bond RL, Russell LL, Dick K, Ariti C, et al. Retained capacity for perceptual learning of degraded speech in primary progressive aphasia and Alzheimer's disease. Alzheimers Res Ther. 2018;10:70. This article reports findings suggesting that perceptual learning for degraded speech is retained across major PPA variants. This heralds a fresh perspective on dynamic physiological processes in PPA emphasising retained language system plasticity.

53. Weil RS, Pappa K, Schade RN, Schrag AE, Bahrami B, Schwarzkopf DS, et al. The Cats-and-Dogs test: a tool to identify visuoperceptual deficits in Parkinson's disease. Mov Disord. 2017;32:1789-90.

54. Marshall CR, Hardy CJD, Russell LL, Bond RL, Sivasathiaseelan $\mathrm{H}$, Greaves C, et al. The functional neuroanatomy of emotion processing in frontotemporal dementias. Brain. 2019.

55. Kumfor F, Hazelton JL, Rushby JA, Hodges JR, Piguet O. Facial expressiveness and physiological arousal in frontotemporal dementia: phenotypic clinical profiles and neural correlates. Cogn Affect Behav Neurosci Springer New York LLC. 2019;19:197-210.

56. Marshall CR, Hardy CJD, Allen M, Russell LL, Clark CN, Bond $\mathrm{RL}$, et al. Cardiac responses to viewing facial emotion differentiate frontotemporal dementias. Ann Clin Transl Neurol. 2018.

57.• Marshall CR, Hardy CJD, Russell LL, Clark CN, Dick KM, Brotherhood EV, et al. Impaired interoceptive accuracy in semantic variant primary progressive aphasia. Front Neurol. 2017;8. This article presents data showing that cardiac responses to facial emotions may differentiate PPA/FTD syndromes. It represents a growing interest in physiological/autonomic markers of PPA in the field at large.

58. Sani TP, Bond RL, Marshall CR, Hardy CJD, Russell LL, Moore $\mathrm{KM}$, et al. Sleep symptoms in syndromes of frontotemporal dementia and Alzheimer's disease: a proof-of-principle behavioural study. eNeurologicalSci Elsevier BV; 2019;17.

59. Bevan-Jones WR, Cope TE, Simon Jones P, Passamonti L, Hong YT, Fryer TD, et al. AV-1451 binding in vivo mirrors the expected distribution of TDP-43 pathology in the semantic variant of primary progressive aphasia. J Neurol Neurosurg Psychiatry BMJ Publishing Group. 2018;89:1032-7.

60. Malpetti M, Passamonti L, Rittman T, Jones PS, Rodríguez PV, Bevan-Jones WR, et al. Neuroinflammation and tau co-localize in vivo in progressive supranuclear palsy. Ann Neurol Wiley; 2020; ana.25911.

61. Bevan-Jones WR, Cope TE, Jones PS, Kaalund SS, Passamonti L, Allinson K, et al. Neuroinflammation and protein aggregation colocalize across the frontotemporal dementia spectrum. Brain NLM (Medline). 2020;143:1010-26.

62.• Benhamou E, Marshall CR, Russell LL, Hardy CJD, Bond RL, Sivasathiaseelan $\mathrm{H}$, et al. The neurophysiological architecture of semantic dementia: spectral dynamic causal modelling of a neurodegenerative proteinopathy. Sci Rep. Nat Res Forum. 2020;10: 16321. This study presents a novel neurophysiological architecture for the erosion of conceptual boundaries in SvPPA, and a new neuroanatomically informed tool (spectral DCM) for analysing network breakdown in PPA.

63. Borghesani V, Hinkley LBN, Ranasinghe KG, Thompson MMC, Shwe W, Mizuiri D, et al. Taking the sublexical route: brain dynamics of reading in the semantic variant of primary progressive aphasia. Brain NLM (Medline). 2020;143:2545-60.

64. Cope TE, Shtyrov Y, MacGregor LJ, Holland R, Pulvermüller F, Rowe JB, et al. Anterior temporal lobe is necessary for efficient lateralised processing of spoken word identity. Cortex Masson SpA. 2020;126:107-18.

65. Ranasinghe KG, Hinkley LB, Beagle AJ, Mizuiri D, Honma SM, Welch AE, et al. Distinct spatiotemporal patterns of neuronal functional connectivity in primary progressive aphasia variants. Brain Oxford University Press. 2017;140:2737-51.

66. Sami S, Williams N, Hughes LE, Cope TE, Rittman T, CoyleGilchrist ITS, et al. Neurophysiological signatures of Alzheimer's disease and frontotemporal lobar degeneration: pathology versus phenotype. Brain Oxford University Press. 2018;141:2500-10.

67. Bonakdarpour B, Rogalski EJ, Wang A, Sridhar J, Mesulam MM, Hurley RS. Functional connectivity is reduced in early-stage primary progressive aphasia when atrophy is not prominent. Alzheimer Dis Assoc Disord. Lippincott Williams and Wilkins. 2017;31:101-6.

68. Friederici AD. The cortical language circuit: from auditory perception to sentence comprehension. Trends Cogn Sci Elsevier Current Trends. 2012:262-8.

69. Johnson J, Marshall C, Weil R, Bamiou D, Hardy C, Warren J. Hearing and dementia: from ears to brain. Brain. 2020; in press.

70. Hardy CJD, Marshall CR, Golden HL, Clark CN, Mummery CJ, Griffiths TD, et al. Hearing and dementia. J Neurol. Springer. Berlin Heidelberg. 2016;263:2339-54.

71. Goll JC, Crutch SJ, Loo JHY, Rohrer JD, Frost C, Bamiou D-E, et al. Non-verbal sound processing in the primary progressive aphasias. Brain. 2010;133:272-85.

72. Hailstone JC, Ridgway GR, Bartlett JW, Goll JC, Buckley AH, Crutch SJ, et al. Voice processing in dementia: a neuropsychological and neuroanatomical analysis. Brain. 2011;134:2535-47.

73. Mahoney CJ, Rohrer JD, Goll JC, Fox NC, Rossor MN, Warren JD. Structural neuroanatomy of tinnitus and hyperacusis in semantic dementia. J Neurol Neurosurg Psychiatry. 2011;82:1274-8.

74.• Hardy CJD, Frost C, Sivasathiaseelan H, Johnson JCS, Agustus $\mathrm{JL}$, Bond RL, et al. Findings of impaired hearing in patients with nonfluent/agrammatic variant primary progressive aphasia. JAMA Neurol. 2019; This study provides evidence for pure tone detection deficits in nfvPPA, underlining both the involvement of 
nonverbal auditory processing and the challenge of interpreting 'peripheral' hearing loss in PPA.

75.• Grube M, Bruffaerts R, Schaeverbeke J, Neyens V, De Weer A-S, Seghers A, et al. Core auditory processing deficits in primary progressive aphasia. Brain Oxford University Press. 2016;139: 1817-29. The authors report on nonverbal auditory processing deficits in PPA syndromes, and present an argument for how these might be linked to speech dysfunction in nfvPPA.

76. Hardy CJD, Agustus JL, Marshall CR, Clark CN, Russell LL, Bond RL, et al. Behavioural and neuroanatomical correlates of auditory speech analysis in primary progressive aphasias. Alzheimer's Res Ther BioMed Central. 2017;9:53.

77. Cope TE, Sohoglu E, Sedley W, Patterson K, Jones PS, Wiggins J, et al. Evidence for causal top-down frontal contributions to predictive processes in speech perception. Nat Commun. 2017;8. The authors use magnetoencephalography to show that degeneration of frontal regions in nfvPPA leads to delayed reconciliation of predictions about degraded speech signals in temporal cortex.

78.•• Johnson JCS, Jiang J, Bond RL, Benhamou E, Requena-Komuro M, Russell LL, et al. Impaired phonemic discrimination in logopenic variant primary progressive aphasia. Ann Clin Transl Neurol WileyBlackwell. 2020;7:1252-7. This article describes phonemic discrimination deficits in IvPPA, with implications for understanding of the mechanism of this syndrome, and involvement of auditory processing in the PPA spectrum more generally.

79. Hardy C, Yong K, Goll J, Crutch S, Warren J. Impairments of auditory scene analysis in posterior cortical atrophy. Brain. 2020; in press.

80. Goll JC, Kim LG, Ridgway GR, Hailstone JC, Lehmann M, Buckley AH, et al. Impairments of auditory scene analysis in Alzheimer's disease. Brain. 2012;135:190-200.

81. Hailstone JC, Crutch SJ, Vestergaard MD, Patterson RD, Warren JD. Progressive associative phonagnosia: a neuropsychological analysis. Neuropsychologia. 2010;48:1104-14.

82. Utianski RL, Duffy JR, Clark HM, Machulda MM, Dickson DW, Whitwell JL, et al. Prominent auditory deficits in primary progressive aphasia: a case study. Cortex Elsevier. 2019.

83. Mesulam M. Slowly progressive aphasia without generalized dementia. Ann Neurol. 1982;11:592-8.

84. Mesulam M-M, Nelson MJ, Hyun J, Rader B, Hurley RS, Rademakers R, et al. Preferential disruption of auditory word representations in primary progressive aphasia with the neuropathology of FTLD-TDP type A. Cogn Behav Neurol. 2019.

85. Watanabe H, Ikeda M, Mori E. Non-fluent/agrammatic variant of primary progressive aphasia with generalized auditory agnosia. Front Neurol Frontiers Media SA. 2020;11:519.

86. Panza F, Lozupone M, Seripa D, Daniele A, Watling M, Giannelli $\mathrm{G}$, et al. Development of disease-modifying drugs for frontotemporal dementia spectrum disorders. Nat Rev Neurol Nature Research. 2020:213-28.

87. Cotelli M, Manenti R, Ferrari C, Gobbi E, Macis A, Cappa SF, Effectiveness of language training and non-invasive brain stimulation on oral and written naming performance in Primary Progressive Aphasia: a meta-analysis and systematic review. Neurosci Biobehav Rev Elsevier Ltd. 2020:498-525.

88. Nissim NR, Moberg PJ, Hamilton RH. Efficacy of noninvasive brain stimulation (tDCS or TMS) paired with language therapy in the treatment of primary progressive aphasia: an exploratory metaanalysis. Brain Sci Multidisciplinary Digital Publishing Institute. 2020;10:597.

89. Bereau M, Magnin E, Nicolier M, Berthet L, Dariel E, Ferreira S, et al. Left prefrontal repetitive transcranial magnetic stimulation in a logopenic variant of primary progressive aphasia: a case report. Eur Neurol S Karger AG. 2016;76:12-8.

90. Di Stasio F, Antonio S, Andrea F, Luca M, Francesco A, Antonella C, et al. Parkinsonism is associated with altered primary motor cortex plasticity in frontotemporal dementia-primary progressive aphasia variant. Neurobiol Aging Elsevier Inc. 2018;69:230-8.

91. Margolis SA, Festa EK, Papandonatos GD, Korthauer LE, Gonsalves MA, Oberman L, et al. Brain Stimul Elsevier Inc. 2019:1340-2.

92. Hartwigsen G, Saur D. Neuroimaging of stroke recovery from aphasia - insights into plasticity of the human language network. Neuroimage Academic Press Inc. 2019:14-31.

93. Kasselimis DS, Simos PG, Peppas C, Evdokimidis I, Potagas C. The unbridged gap between clinical diagnosis and contemporary research on aphasia: a short discussion on the validity and clinical utility of taxonomic categories. Brain Lang Academic Press Inc; 2017;164:63-67.

94. Cope TE, Wilson B, Robson H, Drinkall R, Dean L, Grube M, et al. Artificial grammar learning in vascular and progressive nonfluent aphasias. Neuropsychologia. 2017;104:201-13.

95. Europa E, Iaccarino L, Perry DC, Weis E, Welch AE, Rabinovici GD, et al. Diagnostic assessment in primary progressive aphasia: an illustrative case example. Am J Speech-Language Pathol American Speech Language Hearing Association. 2020:1-17.

96. Schaffer KM, Wauters L, Berstis K, Grasso SM, Henry ML. Modified script training for nonfluent/agrammatic primary progressive aphasia with significant hearing loss: a single-case experimental design. Neuropsychol Rehabil Routledge. 2020:1-30.

97. Jokel R, Kielar A, Anderson ND, Black SE, Rochon E, Graham S, et al. Behavioural and neuroimaging changes after naming therapy for semantic variant primary progressive aphasia. Neuropsychologia Elsevier Ltd. 2016;89:191-216.

98. Croot K. Treatment for lexical retrieval impairments in primary progressive aphasia: a research update with implications for clinical practice. Semin Speech Lang Thieme Medical Publishers, Inc. 2018;39:242-56.

99. Croot K, Raiser T, Taylor-Rubin C, Ruggero L, Ack1 N, Wlasich $\mathrm{E}$, et al. Lexical retrieval treatment in primary progressive aphasia: an investigation of treatment duration in a heterogeneous case series. Cortex Masson SpA. 2019;115:133-58.

100.• Henry ML, Hubbard HI, Grasso SM, Dial HR, Beeson PM, Miller BL, et al. Treatment for word retrieval in semantic and logopenic variants of primary progressive aphasia: immediate and long-term outcomes. 2019; The authors report on a behavioural intervention with lasting and generalized improvement of communication function in people with nfvPPA.

101. Cadório I, Lousada M, Martins P, Figueiredo D. Generalization and maintenance of treatment gains in primary progressive aphasia (PPA): a systematic review. Int J Lang Commun Disord Wiley Blackwell. 2017:543-60.

102. Henry ML, Hubbard HI, Grasso SM, Mandelli ML, Wilson SM, Sathishkumar MT, et al. Retraining speech production and fluency in non-fluent/agrammatic primary progressive aphasia. Brain Oxford University Press. 2018;141:1799-814.

103. Volkmer A, Spector A, Meitanis V, Warren JD, Beeke S. Effects of functional communication interventions for people with primary progressive aphasia and their caregivers: a systematic review. Aging Ment Heal Routledge. 2020;24:1381-93.

104. Kindell J, Sage K, Cruice M. Supporting communication in semantic dementia: clinical consensus from expert practitioners. Qual Ageing Older Adults Emerald Group Publishing Ltd. 2015;16:153-64.

105. Volkmer A, Rogalski E, Henry M, Taylor-Rubin C, Ruggero L, Khayum R, et al. Speech and language therapy approaches to managing primary progressive aphasia. Pract Neurol BMJ Publishing Group Ltd. 2019:practneurol-2018-001921.

106. Volkmer A, Spector A, Warren JD, Beeke S. Speech and language therapy for primary progressive aphasia across the UK: a survey of current practice. Int J Lang Commun Disord Wiley Blackwell. 2019;54:914-26.

107. Volkmer A, Spector A, Warren JD, Beeke S. Speech and language therapy for primary progressive aphasia: referral patterns and 
barriers to service provision across the UK. Dementia SAGE Publications Ltd. 2020;19:1349-63.

108. Taylor-Rubin C, Croot K, Power E, Savage SA, Hodges JR, Togher L. Communication behaviors associated with successful conversation in semantic variant primary progressive aphasia. Int Psychogeriatrics Cambridge University Press. 2017;29:1619-32.

109. Taylor-Rubin C, Azizi L, Croot K, Nickels L. Primary progressive aphasia education and support groups: a clinical evaluation. Am J Alzheimer's Dis Other Dementiasr SAGE Publications. 2020;35: 153331751989563.

110. Pulvermüller F. Neurobiological mechanisms for semantic feature extraction and conceptual flexibility. Top Cogn Sci WileyBlackwell. 2018;10:590-620.

111. Friston K. A theory of cortical responses. Philos Trans R Soc. 2005;360:815-36.

112. Lambon Ralph MA, Sage K, Jones RW, Mayberry EJ. Coherent concepts are computed in the anterior temporal lobes. Proc Natl Acad Sci U S A. 2010;107:2717-22.

113. Jung JY, Williams SR, Sanaei Nezhad F, Lambon Ralph MA. GABA concentrations in the anterior temporal lobe predict human semantic processing. Sci Rep Nature Publishing Group. 2017;7:1-9.

114. Rohrer JD, Rossor MN, Warren JD. Syndromes of nonfluent primary progressive aphasia: a clinical and neurolinguistic analysis. Neurology. 2010;75:603-10.

115. Leyton CE, Ballard KJ, Piguet O, Hodges JR. Phonologic errors as a clinical marker of the logopenic variant of PPA. Neurology Lippincott Williams and Wilkins. 2014;82:1620-7.

116. Caffarra P, Gardini S, Cappa S, Dieci F, Concari L, Barocco F, et al. Degenerative jargon aphasia: unusual progression of logopenic/phonological progressive aphasia? Behav Neurol. 2013;26:89-93.

117. Rohrer JD, Rossor MN, Warren JD. Neologistic jargon aphasia and agraphia in primary progressive aphasia. J Neurol Sci Elsevier. 2009;277:155-9.

118. Teichmann M, Kas A, Boutet C, Ferrieux S, Nogues M, Samri D, et al. Deciphering logopenic primary progressive aphasia: a clinical, imaging and biomarker investigation. Brain Oxford University Press. 2013;136:3474-88.

119. Henry ML, Wilson SM, Babiak MC, Mandelli ML, Beeson PM, Miller ZA, et al. Phonological Processing in primary progressive aphasia. J Cogn Neurosci. 2016;28:210-22.

120. Spitsyna G, Warren JE, Scott SK, Turkheimer FE, Wise RJS. Converging language streams in the human temporal lobe. J Neurosci Society for Neuroscience. 2006;26:7328-36.

121. Leyton CE, Hodges JR, Piguet O, Ballard KJ. Common and divergent neural correlates of anomia in amnestic and logopenic presentations of Alzheimer's disease. Cortex Masson SpA. 2017;86:45-54

122. Warren JE, Wise RJS, Warren JD. Sounds do-able: auditorymotor transformations and the posterior temporal plane. Trends Neurosci. 2005;28:636-43.

123. Hardy CJD, Hwang YT, Bond RL, Marshall CR, Ridha BH, Crutch SJ, et al. Donepezil enhances understanding of degraded speech in Alzheimer's disease. Ann Clin Transl Neurol. 2017.

124. Croot K, Ballard K, Leyton CE, Hodges JR. Apraxia of speech and phonological errors in the diagnosis of nonfluent/agrammatic and logopenic variants of primary progressive aphasia. J Speech Lang Hear Res. 2012;55:S1562.
125. Goll JC, Kim LG, Hailstone JC, Lehmann M, Buckley A, Crutch SJ, et al. Auditory object cognition in dementia. Neuropsychologia Elsevier Ltd. 2011;49:2755-65.

126. Golden H, Clark C, Nicholas J. Music perception in dementia. J Alzheimers Dis. 2017;55:933-49. The authors report on a study of music perception in nfvPPA and IvPPA, relative to patients with typical Alzheimer's disease and healthy control participants. Relative to controls, patients with Alzheimer's disease and IVPPA showed deficits in melody contour processing, while those with nfvPPA showed deficits with local (interval) and global pitch processing.

127. Schaeverbeke J, Bruffaerts R, Grube M, Neyens V, Bergmans B, Dries E. Deficits in rhythm processing in PPA are linked to SMA atrophy. J Neurochem. 2016;1:222-428.

128. Bruffaerts R, Schaeverbeke J, Grube M, Gabel S, De Weer A-S, Dries E, et al. Left frontal white matter atrophy links to timing mechanisms relevant for apraxia of speech. medRxiv. Cold Spring Harbor Laboratory Press. 2019:1.

129. Sivasathiaseelan H, Marshall CR, Hardy CJD, Fox NC, Warren $\mathrm{JD}$, Rossor MN. Aphasic binary reversals in patients with neurological disease as a barrier to clinical decision making. JAMA Neurol American Medical Association. 2019:234-5.

130. Bamiou DE, Musiek FE, Luxon LM. The insula (Island of Reil) and its role in auditory processing: literature review. Brain Res Rev Elsevier. 2003:143-54.

131. Caplan D. Why is Broca's area involved in syntax? Cortex Masson SpA. 2006;42:469-71.

132. Kosillo P, Smith AT. The role of the human anterior insular cortex in time processing. Brain Struct Funct Brain Struct Funct. 2010;214:623-8.

133. Fitch WT, Martins MD. Hierarchical processing in music, language, and action: Lashley revisited. Ann N Y Acad Sci. 2014;1316:87-104.

134. Matchin WGA. neuronal retuning hypothesis of sentencespecificity in Broca's area. Psychon. Bull Rev. 2018:1682-94.

135. Yi HG, Leonard MK, Chang EF. The encoding of speech sounds in the superior temporal gyrus. Neuron. 2019:1096-110.

136. Poeppel D, Assaneo MF. Speech rhythms and their neural foundations. Nat Rev Neurosci Nature Research. 2020:322-34.

137. Buchsbaum BR, D'Esposito M. A sensorimotor view of verbal working memory. Cortex. 2019:134-48.

138. Pulvermüller F. Neural reuse of action perception circuits for language, concepts and communication. Prog Neurobiol. 2018:1-44.

139. Tao Y, Ficek B, Rapp B, Tsapkini K. Different patterns of functional network reorganization across the variants of primary progressive aphasia: a graph-theoretic analysis. Neurobiol Aging. 2020;96:184-96.

140.• Suárez-González A, Zimmermann N, Waddington C, Wood O, Harding E, Brotherhood E, et al. Non-memory led dementias: care in the time of covid-19. BMJ; 2020. The authors put forward a callto-action for adapted management of PPA and other rare dementias with challenging needs in the Covid-19 era.

141. Dial HR, Hinshelwood HA, Grasso SM, Hubbard HI, GornoTempini ML, Henry ML. Investigating the utility of teletherapy in individuals with primary progressive aphasia. Clin Interv Aging. 2019;14:453-71.

Publisher's Note Springer Nature remains neutral with regard to jurisdictional claims in published maps and institutional affiliations. 